\title{
Temperature dependence of Self-diffusion coefficient (SDC) of liquid aluminum using Embedded Atom Method-Finnis-Sinclair (EAMFS) Potential
}

\author{
*NENUWE, ON; OSAFILE, OE \\ Department of Physics, Federal University of Petroleum Resources, \\ P. M. B. 1221, Effurun, Delta State, Nigeria. *Email address: nenuwe.nelson@fupre.edu.ng; Phone: +2348037295834
}

\begin{abstract}
Atomistix tool kit force field calculations have been done, using the embedded atom method-FinnisSinclair potential as implemented in Virtual Nano Lab-Atomistix Tool Kit (VNL-ATK) to study the temperature dependence of self-diffusion coefficient of liquid aluminum. Also, we calculated the shear viscosity from the SutherlandEinstein relation, and reports that, the calculated SDC and viscosity are sensitive to temperature. Results obtained at 980 , 1020 and $1060 \mathrm{~K}$ for SDC underestimated available experimental results, while the calculated results for viscosity at 1000 and $1200 \mathrm{~K}$ overestimated experimental data available in the literature. The underestimation might be due to the shortrange order in liquid $\mathrm{Al}$.
\end{abstract}

DOI: https://dx.doi.org/10.4314/jasem.v22i4.14

Copyright: Copyright $\odot 2018$ Nenuwe and Osafile. This is an open access article distributed under the Creative Commons Attribution License (CCL), which permits unrestricted use, distribution, and reproduction in any medium, provided the original work is properly cited.

Dates: Received: 12 March 2018; Revised: 09 April: 2018; Accepted: 20 April 2018

Keywords: self-diffusion, viscosity, aluminum, temperature.

The thermophysical property of self-diffusion plays a significant role in metallurgical processes and solidification. Therefore, the knowledge of accurate self-diffusion coefficient is thus crucial for atomistic simulation methods and refining casting techniques for the synthesis of new materials (Kurz and Fisher, 1986; Sandro and Zach, 2017). Experimentally, the results for diffusion coefficients in liquid metals usually show deviations up to 100\% (Banish and Lyle, 1999). According to Meyer (2010), SDCs are normally overestimated in the range of $10 \%-100 \%$ and also their temperature dependence deviates from the actual value without convection. It is also well known in the literature that the use of different potential functions gives slightly varying results for the self-diffusion coefficient, and the data for liquid metals are scarce and contains large errors (Merher, 2007; Ju et al., 2013). However, Ju et al., (2013), in their work, did a study of self-diffusion coefficients for liquid metals $(\mathrm{Cu}$ and $\mathrm{Al})$ by using the embedded atom method (EAM) potential function. The results of self-diffusion coefficient for liquid copper and aluminum show inverse square relationship between the natural logarithm of self-diffusion coefficients and temperature and that diffusion coefficient increases with temperature. Their results for $\mathrm{Cu}$ and $\mathrm{Al}$ are in close agreement with results simulated by MKBA1 that are close to experimental values (Kargl et al., 2013). Also, Cherne and Deymier (2001), studied the shear viscosity and SDC of liquid aluminum (Al) by using the Equilibrium Molecular Dynamics (MD) and
Non-Equilibrium Molecular Dynamics with the EAM potential function. Their results agree with the Sutherland-Einstein model, but the temperature dependence disagrees with the universal scaling law (Dzugutov, 1996). In a recent experimental work by Kargl et al., (2013), they did a measurement of selfdiffusion coefficient of liquid $\mathrm{Al}$ by the incoherent Quasielastic Neutron Scattering (QNS) at different temperatures and found that the aluminum SDCs also increased with temperature and follow the Arrhenius law with activation energy of $280 \pm 70 \mathrm{meV}$. Noel and Alain (2013), in their work, did a study of dynamic properties of liquid aluminum using DFT within LDA and GGA approximations. They found that the selfdiffusion coefficient results obtained from GGA underestimate both LDA based results and experimental results. Alfe and Gillan (1998) used density functional theory within LDA approximation to calculate the self-diffusion and viscosity coefficients and showed that the SDC and viscosity results are in the range of $(5.2-6.8) \times 10^{-9} \mathrm{~m}^{2} / \mathrm{s}$, and $(1.4-6.8) \times 10^{-3}$ Pa.s, respectively. These results underestimate experimental data for SDC and overestimates experimental values for viscosity coefficient.

Therefore, we studied the self-diffusion coefficient of liquid aluminum (Al) using $\mathrm{MD}$ technique based on the embedded atom method-Finnis-Sinclair potential function (Jess and Brian, 2000) as implemented in Virtual Nano Lab-Atomistix Tool Kit (Atomistix 
Toolkit, 2017), and the shear viscosity is calculated from the Sutherland-Einstein relation. This paper focuses on the temperature dependence of selfdiffusion coefficient and shear viscosity of liquid aluminum.

\section{MATERIALS AND METHODS}

The system is prepared with a face-centered cubic crystal structure of aluminum in space group 225 Fm$3 \mathrm{~m}$ (Kings, 2002), using periodic boundary conditions on a supercell size of $16 a_{0} \times 16 a_{0} \times 16 a_{0}$ (where the lattice constant $a_{0}=4.04958 \AA$ ) with 16,384 atoms. The calculations were performed using ATK-force field code (Atomistix Toolkit, 2017; Griebel et al., 2007; Griebel and Hamaekers 2004), together with the EAMFS potential (Jess and Brian, 2000; Sheng et al., 2011; Daw and Baskes, 1984) as implemented in the Virtual Nano Lab 2017.2 (Atomistix Toolkit, 2017). Usually, in classical MD simulations, particles are moved by using forces extracted from semi-empirical interatomic potentials (Noel and Alain, 2013). Therefore, the potential function plays key role in MD simulations. The potential energy ( $\mathrm{Ju}$ et al., 2013) is defined as

$$
\begin{aligned}
& E_{t o t}=\frac{1}{2} \sum_{i \neq j} V\left(r_{i j}\right)+\sum_{i} F\left(\rho_{i}\right) \\
& \rho_{i}=\sum_{j} \phi\left(r_{i j}\right)
\end{aligned}
$$

Where $E_{\text {tot }}$ is the total energy, $V\left(r_{i j}\right)$ is the potential function, $\phi\left(r_{i j}\right)$ is the electron density contribution from atom $j$ to atom $i, F\left(\rho_{i}\right)$ is the embedding function and $\rho_{i}$ is the total electron density at an atomic position $i$ calculated through the linear superposition of electron density contributions from neighboring atoms.

The supercell structure of crystalline $\mathrm{Al}$ was first heated above its melting point (933K) for 100,000 steps at intervals of 2000 with a time integration step of 1 femto second (fs) at a fixed pressure of 1 bar using the constant number of particles, pressure and temperature (NPT) Martyna Tobias Klein barostat. This allows one to increase the temperature of $\mathrm{Al}$ crystal gradually above its melting point (from $800-$ 933.5K). It implies that the ordered solid aluminum will eventually become a liquid that only exhibits a short-range order. Next is the annealing of liquid aluminum to bring the system to equilibrium. The supercell structure of liquid $\mathrm{Al}$ is equilibrated at constant temperature $933.5 \mathrm{~K}$, using the same NPT for 100,000 steps with Maxwell-Boltzmann velocity type. This helps to eliminate any memory effect of the initial solid aluminum on the physical properties of liquid aluminum obtained from the initial supercell structure. Thirdly, the equilibrated liquid $\mathrm{Al}$ is annealed for another 100,000 steps using the velocities from the second MD simulation as initial velocities. In this step, we used the supercell of the equilibrated liquid aluminum at a constant temperature of $933.5 \mathrm{~K}$, to collect sufficient statistical data for calculating the self-diffusion coefficient of liquid aluminum. The entire process is repeated for a broad temperature range above the melting point.

\section{RESULT AND DISCUSSION}

Figure 1 displays the radial distribution function $g(r)$ simulated for liquid aluminum using the EAMFS potential. The self-diffusion coefficient, $D$ can be calculated from the mean-square displacement (MSD) of the atoms in molecular dynamics simulation (Quantumwise, 2017):

$D=\frac{1}{6 t}\left\langle X^{2}(t)\right\rangle$,

where $\left\langle X^{2}(t)\right\rangle$, is the MSD of aluminum atoms in liquid aluminum, calculated at the observation time $t$.

$$
\left\langle X^{2}(t)\right\rangle \approx \frac{1}{T_{M D}-t} \int_{0}^{T_{M D}-t} \frac{1}{N} \sum_{j=1}^{N}\left[r_{j}\left(t^{\prime}+t\right)-r_{j}\left(t^{\prime}\right)\right]^{2} d t^{\prime}
$$

and $N$ is the total number of aluminum atoms in the supercell, $r_{j}\left(t^{\prime}+t\right)$ and $r_{j}\left(t^{\prime}\right)$ are atomic position coordinates at time $t^{\prime}+t$ and $t^{\prime}$, with $j=1,2, \ldots, N$, and $t$ is the time interval of observation that is adopted to extract the atomic diffusion coefficient from the time-dependent MSD assuming Eq. (4) shows a linear behavior with respect to $t$. The observation time, $t$ is always less than the total simulation time $\left(T_{M D}\right)$ of the MD simulation of liquid. For values of $t$ close to $T_{M D}$, the MSD becomes noisy since it is no longer linearly dependent on $t$. Therefore, for accurate calculation of self-diffusion coefficient only simulations with values of $t$ much smaller than the simulation time $T_{M D}$ are considered.

From Eq. (4), the atomic SDC is averaged over all the aluminum atoms with the assumption that all the $\mathrm{Al}$ atoms are identical, with the same atomic mass and other physical characteristics that may affect diffusion of atoms. As the ordered solid Al transforms into a liquid phase, a short-range order is observed to be preserved on melting. This can be seen in the radial distribution function $g(r)$ Figure 1(a). It shows a welldefined peak around $2.77 \AA$, suggesting that this is the dominant nearest neighbor distance between atoms in liquid aluminum at the various temperatures. 

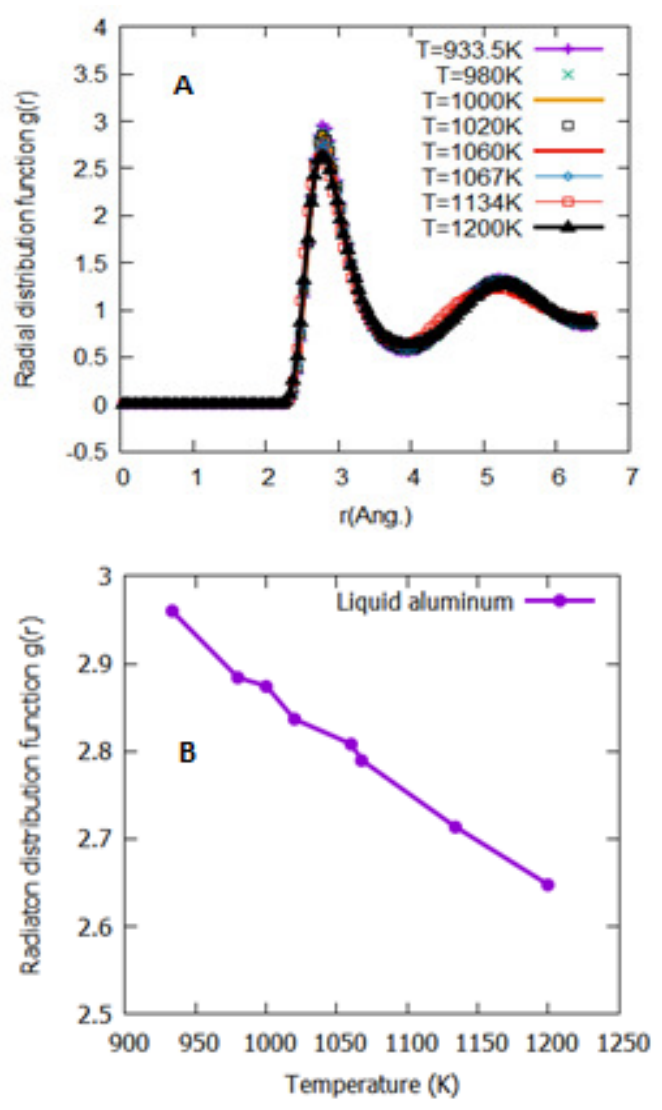

Fig 1. (a) Radial distribution function $g(\mathrm{r})$ for liquid $\mathrm{Al}$ at different temperatures and (b) Temperature dependence of $g(\mathrm{r})$ for liquid aluminum.

Also, we observed that the radial distribution function is temperature dependent and this is reported in Figure 1(b). It decreases from $2.9594 \AA$ to $2.6478 \AA$ as temperature is systematically increased from 933.5 to $1200 \mathrm{~K}$. This implies that as the temperature keeps increasing the atoms in liquid $\mathrm{Al}$ gradually becomes disorderly with respect to their orientation.

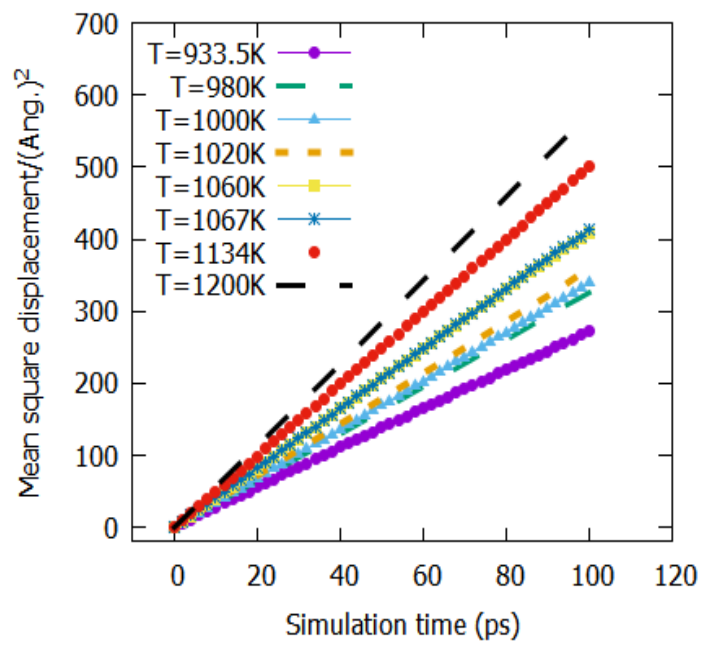

Fig 2. Mean square displacement for liquid $\mathrm{Al}$ at temperature ranging from $933.5 \mathrm{~K}$ to $1200 \mathrm{~K}$.

Figure 2 shows the simulation profile of mean-square displacement at different temperatures from $933.5 \mathrm{~K}$ to $1200 \mathrm{~K}$ based on the EAMFS potential function. It is observed that the MSD varies linearly with time in the whole simulation range of temperatures. The slope of the lines gives the respective self-diffusion coefficient at different temperatures and it increases with temperature as reported in Table 1. This signifies that the metallic aluminum has completely melted. Increasing the temperature from $933.5 \mathrm{~K}$ to $1200 \mathrm{~K}$ resulted in increased self-diffusion coefficient because the atoms move more radically at higher temperatures than at lower temperatures. Results for the selfdiffusion coefficients $D$ obtained in this study are compared with experimental values as shown in Table 1.

Table 1. Calculated self-diffusion coefficients $D$, radial distribution $g(\AA)$, shear viscosity $\eta$, and $r(\AA)$ for Liquid Al compared with experimental results.

\begin{tabular}{ccccccc}
\hline $\mathrm{T}(\mathrm{K})$ & \multicolumn{2}{c}{$D\left(10^{-9} \mathrm{~m}^{2} / \mathrm{s}\right)$} & $g(\AA)$ & $r(\AA)$ & \multicolumn{2}{c}{$\eta(\mathrm{mPa} . \mathrm{s})$} \\
\cline { 2 - 3 } \cline { 5 - 6 } & Present work & Experiment & & & Present work & Experiment \\
933.5 & 4.53 & - & 2.9594 & 2.77 & 1.635 & - \\
980 & 5.44 & $6.6^{\text {(a) }}$ & 2.8838 & 2.77 & 1.429 & - \\
1000 & 5.64 & - & 2.8744 & 2.77 & 1.406 & $1.178^{(\mathrm{b})}$ \\
1020 & 5.95 & $7.2^{\text {(a) }}$ & 2.8366 & 2.77 & 1.362 & - \\
1060 & 6.87 & $8.1^{\text {(a) }}$ & 2.8083 & 2.77 & 1.223 & - \\
1067 & 6.91 & - & 2.7894 & 2.77 & 1.224 & - \\
1134 & 8.35 & - & 2.7138 & 2.77 & 1.077 & - \\
1200 & 9.59 & - & 2.6478 & 2.77 & 0.996 & $0.865^{(\mathrm{b})}$ \\
\hline \multicolumn{5}{c}{${ }^{(\mathrm{a})}\left(\right.$ Kargl et al., 2013), ${ }^{(\mathrm{b})}$ (Assael et al., 2002) }
\end{tabular}

(Kargl et al., 2013), LDA and LDA-AIMD results by

At simulation temperatures 980, 1020 and $1060 \mathrm{~K}$, our results underestimated the experimental values by $17.58 \%, 17.36 \%$ and $17.08 \%$, respectively. This trend has earlier been established (Noel and Alain, 2013), where GGA results underestimated experimental
20\% (Alemany et al., 2004; Alfe and Gillan, 1998). The temperature dependence of $D$ using the EAMFS potential as reported in Figure 3(a) shows that the selfdiffusion coefficient increases with increasing temperature. This agrees with the Arrhenius law (Ju et al., 2013). We can see how the experimental results 
obtained by Kargl et al. (2013) are underestimated by results from this study.

It is well known that the Stokes-Einstein and Sutherland-Einstein relations relate the self-diffusion coefficient to the shear-viscosity of liquids (Kargl et al., 2013; Einstein, 1905). Therefore, we also calculated the shear-viscosity of liquid aluminum using the Sutherland-Einstein relation.

$$
\eta=\frac{k_{B} T}{4 \pi r D}
$$

Where $\eta$ is the shear-viscosity, $D$ is the self-diffusion coefficient, $k_{B}$ is Boltzmann constant, $r$ is radius of the moving particle and $T$ is the absolute temperature. It well known (Noel and Alain, 2013) that the effective atomic diameter of moving particles is taken as the position of the first peak in the radial distribution function. At all temperatures, the first peak position is found to be $2.77 \AA$ as reported in Table 1 and Figure 1. This implies that the atomic radius used for the current study is $r=1.385 \AA$.

Results obtained from Eq. (5) are reported in Table 1 and Figure 3(b). It is clear in Figure 3(b) that the shearviscosity of liquid aluminum decreased with increasing temperature. When compared with experimental data (Assael et al., 2002), we found that the results at $1000 \mathrm{~K}$ and $1200 \mathrm{~K}$ overestimated the experimental results for shear viscosity by $19.3 \%$ and $15.1 \%$ respectively. This trend agrees with other researchers (Noel and Alain, 2013; Alfe and Gillan, 1998; Gonzalez et al., 2002; Ju et al., 2013; Kargl et al., 2013; Cherne and Deymier; 2001; Dzugutov, 1996; Noel and Alain, 2013). Since the shear-viscosity coefficient is inversely proportional to the selfdiffusion coefficient, it is expected that it should decrease with increasing temperature. This response to temperature change has great applications in the processing of crystal formation and rate of transfer of impurity elements from metal to slag during extractive metallurgy.

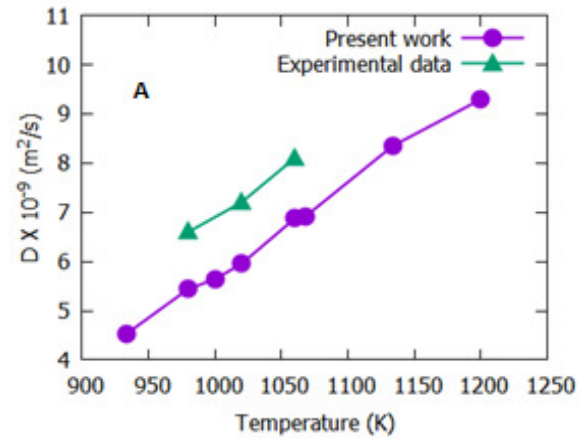

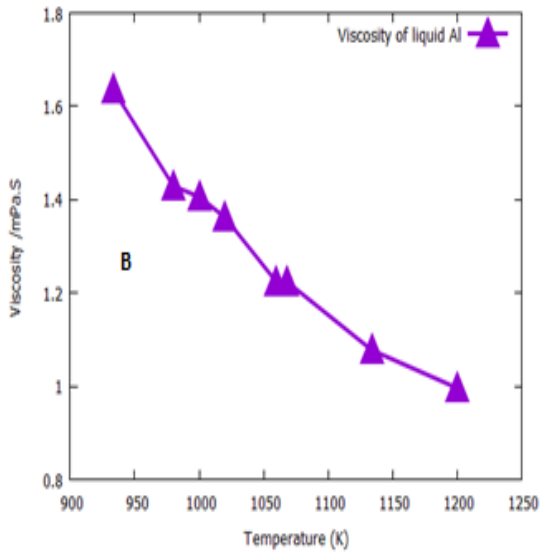

Fig 3. Temperature dependence of (a) Self-diffusion coefficient D for liquid Al calculated with EAMFS potential compared with experimental data, (b) Shear-viscosity of liquid $\mathrm{Al}$ calculated from the Sutherland-Einstein relation.

Conclusion: We have calculated the self-diffusion coefficients of liquid Al using EAMFS and ATK-force field code at different temperatures. Results obtained are found to be sensitive to temperature. The selfdiffusion coefficient is observed to increase with increasing temperature because the atoms in liquid $\mathrm{Al}$ move more radically at higher temperatures. Also, the calculated viscosity for liquid Al decreases with increasing temperature. The behavior of these thermophysical properties in response to change in temperature has great applications in the metallurgical industry.

Acknowledgement: The authors are grateful to the Federal University of Petroleum Resources Effurun for providing the Mini Workstation used in the calculations and also to Quantumwise for access to use the VNL-ATK software package for the simulation process. We also wish to appreciate the Marie Curie Library of the Abdus Salam International Centre for Theoretical Physics (ICTP) for permission to use the electronic journal delivery service (ejds) for the reference articles in this publication.

\section{REFERENCES}

Alemany, MMG; Gallego, LJ; Gonzalez, DJ (2004). Kohn-Sham ab initio Molecular Dynamics study of Al near Melting. Phys. Rev. B 70: 134206-1 134206-6.

Alfe, D; Gillan MJ (1998). First-Principle Calculations of Transport Coefficients. Phys. Rev. Lett. 81: $5161-5164$.

Assael, MJ; Banish, RM; Engry, I; Brill, J Brooks, R; Quested, PN; Mills, KC; Akira, N; Sato, Y; Wakeham, WA(2002). Reference Data for 
Density, and Viscosity of Liquid Aluminum and Iron. J. Phys. Chem. Ref. Data 35 (1): 285 - 300.

Atomistix ToolKit 2017.2. Quantumwise A/S. www.quantumwise.com, 2017.2.

Banish, RM and Lyle BJ (1999). In-situ diffusivity measurement technique. Adv. Space Res. 24: 1311-1320.

Cherne, FJ; Deymier, PA (2001). Calculation of the Transport Properties of Liquid Aluminum with Equilibrium and Non-equilibrium Molecular Dynamics. Scripta Materialia 45: 985-991.

Daw, MS; Baskes, MI (1984). Embedded-atom method: Derivation and Application to Impurities, Surfaces, and other Defects in metals. Phys. Rev. B 29 (12): 6443 - 6453.

Dzugutov, M (1996). A Universal Scaling Law for Atomic Diffusion in Condensed Matter. Nature 381: 137.

Einstein, A (1905). Annalen der Physik .17: 549.

Griebel, M; Hamaekers, J (2004). Molecular dynamics simulations of the elastic moduli of polymercarbon nanotube composites. Computer Methods in Appl. Mech. and Eng. 193; 17-20: 1773 - 1788.

Griebel, M; Knapek, S; Zumbusch, G (2007). Numerical Simulation in Molecular Dynamics. Springer.

In CRC Handbook of Chemistry and Physics 83, by Kings, HW, 19. 2002.
Jess, BS; Brian, BL (2000). Adjusting the melting point of a model system via Gibbs-Duhem integration: Application to a model of aluminum. Phys. Rev. B 62, 22: 14720.

Ju, Y; Zhang, Q; Gong, Z; Ji, G (2013). Molecular Dynamics Simulation of Self-Diffusion Coefficients for Liquid Metals. Chin. Phys. B 22, 8: 083101 .

Kargl, F; Weis, H; Unruh, T; Meyer, A (2013). Selfdiffusion in Liquid Aluminum. J. Phys. Conf. Ser. 340: 012077.

Kurz, W; Fisher, DJ (1996). Fundamentals of Solidification. Aedermannsdorf: Trans-Tech.

Merher, H (2007). Diffusion in solids. Heidelberg: Springer.

Meyer, A (2010). Self-diffusion in Liquid Copper as seen by Quasielastic Neutron Scattering. Phys. Rev. B 81: 012102-1 - 012102-3.

Noel, J; Alain, P (2013). Liquid Aluminum: Atomic Diffusion and Viscosity from ab initio Molecular Dynamics, Scientific Report. 3: 3135.

Quantumwise Document. www.quantumwise.com, 2017.

Sandro, S; Zach, E (2017). Self-diffusion in liquid AlGe investigated with Quasi-elastic Neutron Scattering. Appl. Phys. Lett. 110: 161903.

Sheng, HW; Kramer, MJ; Cadien, A; Fujita, T; Chen, MW (2011). Highly optimized embedded-atommethod potentials for fourteen fcc metals. Phys. Rev. B 83: 134118. 